\title{
A percepção dos alunos do mestrado em engenharia de produção sobre a existência de ambientes de criação do conhecimento
}

\author{
Lucyanno Moreira Cardoso Holanda \\ Graduado em administração pela Universidade Federal de Campina Grande, aluno do mestrado da Universidade Tecnológica Federal do Paraná. \\ E-mail: lucyanno@gmail.com
}

\begin{abstract}
Antonio Carlos de Francisco
Doutor em engenharia de produção pela Universidade Federal de Santa Catarina. Mestre em tecnologia pela Universidade Tecnológica Federal do Paraná. Professor dos programas de pós-graduação em engenharia de produção e de ensino de ciência e tecnologia da UTFPR.

E-mail: acfrancisco@utfpr.edu.br
\end{abstract}

João Luiz Kovaleski

Doutor pela Universite Joseph Fourier de Grenoble - UJFG / França. Mestre em automação industrial pela UTFPR. Engenheiro industrial eletrônico pela UTFPR.

E-mail: kovaleski@utfpr.edu.br

\section{Resumo}

Este estudo pautou-se em responder ao seguinte problema: "Como facilitar a emergência de contextos favoráveis ao processo de criação e compartilhamento de conhecimento?" Ante a formulação inicial, o presente artigo tem por objetivo identificar os quatro tipos de contextos (originating ba, dialoguing ba, systemizing ba e exercising ba) e apontar seus benefícios para a criação e compartilhamento de conhecimentos, a partir da percepção dos mestrandos dos cursos de engenharia de produção da Universidade Tecnologica Federal do Paraná (UFTPR) campus Ponta Grossa, de acordo com a metodologia proposta por Nonaka, Toyama e Konno (2002). O texto caracteriza-se como um estudo de caso, com a utilização de técnicas qualitativas para coleta e análise dos dados. Os resultados apontam a identificação dos espaços por $100 \%$ dos respondentes, tendo como benefícios o compartilhamento de informações e de conhecimentos. As informações compartilhadas que mais trouxeram benefícios estão relacionadas à criação e desenvolvimento de artigos, seminários, qualificação e defesa da dissertação.

\section{Palavras-chave}

Contextos favoráveis. Gestão do conhecimento. Criação e compartilhamento do conhecimento.

\section{The perception of production engineering master degree students about the existence of creation environment of knowledge}

\begin{abstract}
This work aims to answer the following initial problem: how to facilitate the emerging of favorable contexts to the process of knowledge creating and sharing? Facing the formulation of the initial hypothesis, the objective in this article is to identify the four type of contexts (originating ba, dialogue ba, systematizing ba e exercising ba) and to point out its benefits for knowledge creation and sharing from the perception of the Production Engineering masters students of UFTPR, campus Ponta Grossa, according to the methodology proposed by Nonaka, Toyama and Konno (2002). This article is characterized as a case study, using qualitative techniques for data collect and analysis. The results point to the space identification by $100 \%$ of the answers is having as benefits the information and knowledge sharing. The shared information that brought more benefits are related to articles creation and development, seminars, qualify and dissertation presentation.
\end{abstract}

\section{Keywords}

Favourable contexts. Knowledge management. Knowledge creation and sharing. 


\section{INTRODUÇÃO}

O atual contexto organizacional é caracterizado pela mutação constante, concorrência cada vez mais acirrada e a necessidade de as organizações buscarem os mecanismos e ações mais adequadas para o alcance de maior competitividade.

Nessa perspectiva, o novo parâmetro para análise organizacional tem sido o conhecimento, o qual pode ser gerenciado, buscando a sua adequação para a estrutura, formas de funcionamento e as estratégias empresariais.

Diante desse contexto inicial, o presente trabalho coloca em pauta para posterior resposta o seguinte problema: Como facilitar a emergência de contextos favoráveis ao processo de criação e compartilhamento de conhecimento? Como resposta ao questionamento, os autores Nonaka e Takeuchi (1997) enfatizam que as condições favoráveis para criação do conhecimento organizacional passam pelo processo chamado SECI (socialização, externalização, combinação e internalização) e necessitam de um contexto físico, virtual e mental, a que os japoneses denominam $\mathrm{B} a$. A partir dele o conhecimento é criado, compartilhado e utilizado.

Partindo da formulação do problema inicial, o presente artigo tem por objetivo identificar os quatro tipos de contextos (originating ba, dialoguing $b a$, systemizing ba e exercising $b a$ ) e apontar seus benefícios para a criação e o compartilhamento de conhecimentos, a partir da percepção dos alunos do mestrado do curso de engenharia de produção da Universidade Tecnológica Federal do Paraná Campus Ponta Grossa de acordo com a metodologia proposta por Nonaka, Toyama e Konno (2002). O artigo caracteriza-se como um estudo de caso, com a utilização de técnicas qualitativas para coleta e análise dos dados.

Além desta parte inicial que contextualiza a gestão do conhecimento (GC) e a necessidade da criação do conhecimento, são abordados no referencial teórico os conceitos principais de GC, o modelo SECI (socialização, externalização, combinação e

Ci. Inf., Brasília, v. 38, n. 2, p. 96-109, maio/ago. 2009 internalização), solicitude nas organizações e os espaços de criação do conhecimento Ba (Originating ba, Dialoguing ba Systemizing ba e Exercising ba).

Em seguida são explicitados os procedimentos metodológicos para coleta e análise dos dados. Depois disso, são apresentados os resultados da pesquisa. E, finalmente, são feitas as considerações finais, mencionando a necessidade da realização de outros estudos para confirmar base conceitual e conhecer como em outros contextos organizacionais são aplicados os mecanismos da gestão do conhecimento.

\section{GESTÃO DO CONHECIMENTO}

A gestão do conhecimento é um conceito que surgiu no final da década de 80 , com o objetivo de promover ("gerenciar") o conhecimento como um recurso organizacional para obtenção de vantagem competitiva.

É um tema que vem ganhando espaço tanto no campo acadêmico quanto no organizacional, pois transforma o conhecimento individual em conhecimento organizacional, inserindo-o em produtos e serviços.

Existem várias formas de abordagens conceituais para a gestão do conhecimento, com destaque para:

- abordagem de Wiig (1993), baseada nas práticas de exploração do conhecimento e sua adequação a partir de práticas de gestão específicas;

- abordagem de Leornard-Barton (1995), foco em atividades que envolvem: 1) busca de soluções criativas, de forma compartilhada; 2) implementação e integração de novas metodologias e ferramentas nos processos atuais; 3) prática de experimentos, a partir de protótipos e projetos-piloto para desenvolvimento de competências; 4) importação e absorção de metodologias e tecnologias externas;

\section{- abordagem de Nonaka e Takeuchi (1997),}

Baseada na transformação do conhecimento explícito em conhecimento tácito e vice-versa, a partir das práticas de socialização (tácito para 
tácito), externalização (tácito para explícito), combinação (explícito para explícito) e internalização (explícito para tácito);

- abordagem de Barclay e Murray (1997), ênfase em aspectos culturais e de redefinição de processos;

- abordagem de Von Krogh, Ichijo e Nonaka (2001), baseada nos capacitadores do conhecimento: (1) instilar a visão do conhecimento, (2) gerenciar conversas, (3) mobilizar os ativistas do conhecimento, (4) criar um contexto adequado e (5) globalizar o conhecimento local. Além disso, os autores explicitam a necessidade de haver solicitude entre as pessoas e estratégias focadas para o conhecimento;

- abordagem de Nonaka, Toyama e Konno (2002), ênfase nas condições favoráveis para criação do conhecimento organizacional, essas condições necessitam de um ambiente físico, virtual e mental, os quais os japoneses denominam $B a$;

- abordagem de Probst, Raub e Romhardt (2002), aplicação de gestão do conhecimento com base na abordagem de "elementos construtivos";

- abordagem de Choo (2003), baseada na organização do conhecimento a partir do uso estratégico da informação.

Este estudo envolve a identificação dos espaços organizacionais mais adequados para a gestão do conhecimento e, mais especificamente, capazes de criar e potencializar o uso mais intensivo das informações e do conhecimento.

Deste modo, optou-se pela abordagem de Nonaka, Toyama e Konno (2002), considerando que os autores exploram de forma mais específica os espaços e os processos de criação do conhecimento.
O modelo SECI (socialização, externalização, combinação e internalização)

O conhecimento organizacional é constituído por dois componentes principais: as formas de interação do conhecimento e os níveis de criação do conhecimento (NONAKA; TAKEUCHI, 1997).

Essas formas de interação entre o conhecimento tácito e conhecimento explícito e entre indivíduo e organização resultarão em quatro processos de conversão do conhecimento que constituem o modelo SECI (espiral do conhecimento), de acordo com a figura 1.

Segundo Nonaka e Takeuchi (1997), os quatro processos de conversão do conhecimento são dependentes, porém cada um possui características, essas são:

- socialização (tácito para tácito) é o processo de compartilhamento de experiências que resulta na criação do conhecimento tácito, como modelos mentais ou habilidades técnicas compartilhadas. Sem alguma forma de experiência compartilhada, é quase impossível uma pessoa interpretar o raciocínio do outro;

- externalização (tácito para explícito) baseiase na formulação do conhecimento explícito pelo 
compartilhamento do conhecimento tácito. Este é traduzido para explícito por meio do uso de palavras e/ou imagens, diálogo, reflexão coletiva, metáforas, analogias e hipóteses, além da dedução, indução e abdução. Segundo Nonaka e Takeuchi (1997), dos quatro modos de conversão do conhecimento esta fase é a mais importante, pois cria conceitos novos e explícitos para as organizações;

- combinação (explícito para explícito): o conhecimento é fundamentado na análise do conhecimento codificado em documentos, memorandos, redes de comunicação computadorizadas, conversas ao telefone, banco de dados e etc. É um processo de estruturação de conceitos em um sistema de conhecimento, envolve a combinação de conjuntos diferentes de conhecimento explícito, e ocorrem troca e combinação de conhecimentos por meios como documentos, reuniões ou redes de comunicação computadorizadas;

- internalização (explícito para tácito) é o processo de incorporação do conhecimento explícito no conhecimento tácito. É intimamente relacionado ao "aprender fazendo". Quando são internalizadas nas bases do conhecimento tácito dos indivíduos sob a forma de modelos mentais ou know-how técnico compartilhado, as experiências, com a socialização, externalização e combinação, tornam-se ativos valiosos. Esse conhecimento tácito acumulado precisa ser compartilhado com outros membros da organização, iniciando assim nova espiral de criação do conhecimento.

Explicitadas as quatro fases de criação do conhecimento, é possível entender que o conhecimento organizacional é criado mediante interação contínua e dinâmica, e essa interação é moldada por um aspecto fundamental, as relações de confiança (solicitude).

\section{Solicitude nas organizações}

As organizações vivem em ambientes de hipercompetição, nos quais necessitam encontrar meios de obter vantagens competitivas sobre seus concor- rentes a todo o momento. Consequentemente, a alta gerência, os sistemas, as estratégias e a estrutura das empresas passam a seguir essa postura. Com efeito, os trabalhadores são afetados em seus sistemas de remuneração e posição, causando possível diminuição nos níveis de confiança e cooperação.

Uma das alternativas que possibilita bons níveis de solicitude entre as pessoas é a criação dos ambientes do conhecimento " $B a$ ”. É necessário lembrar que a solicitude pode existir nas organizações sem a necessidade da criação do $B a$, mas estes não podem prosperar sem a presença da solicitude, pois o conhecimento tácito das pessoas, base para todo conceito da criação, é suscetível às barreiras impostas pela sua ausência.

Para compartilhar o conhecimento pessoal, os indivíduos devem confiar no que os outros estejam dispostos a ouvir e a reagir às suas ideias (VON KROGH; ICHIJO; NONAK, 2001). Os bons relacionamentos diminuem a desconfiança, o medo e a insatisfação, proporcionando possibilidades de exploração de ambientes desconhecidos como novos mercados, novos produtos e novas tecnologias.

Segundo Sabel (1991), a confiança nunca poderá intencionalmente ser criada, e sim gerada a partir de uma estrutura ou de contextos adequados. Já Perrow (1992) argumenta que, embora a confiança não possa ser criada, ela poderá ser encorajada por uma estrutura ou por um contexto que podem ser deliberadamente criados.

Portanto, a solicitude trata de relações de ajuda mútua ao aprendizado, contribuição de conscientização das oportunidades, além de suas consequências, e de fomentar conhecimentos pessoais e compartilhar seus insights.

Para Von Krogh, Ichijo e Nonak (2001), dentro de uma organização, os relacionamentos podem existir em basicamente dois níveis - alta solicitude (tranferência e convivência) e baixa solicitude (captura e transação). 
A alta solicitude é caracterizada por grande confiança, empatia ativa, acesso à ajuda, leniência no julgamento e coragem entre os membros. As pessoas criam conhecimento individual mediante transferência de insights, e os grupos geram o conhecimento social por meio da convivência.

No que se refere à baixa solicitude, tais comportamentos são quase ausentes. A criação do conhecimento individual ocorre por meio de captura, e boa parte do conhecimento social, por meio de transação. A figura 2 resume esses processos.

A partir do conteúdo aqui explicitado, pode-se inferir que a criação do conhecimento organizacional é um processo complexo que envolve um conjunto de variáveis tecnológicas, estruturais e principalmente de ordem sociocomportamental, com implicações múltiplas nas formas de funcionamento da empresa e nas posturas e ações das pessoas.

No entanto, esse processo pode ser facilitado quando as organizações oferecem condições para a criação do "B $a$ ", proporcionando um ambiente capaz de disseminar e compartilhar a informação para todos os setores e pessoas, condição esta imprescindível para que as organizações encontrem as formas devidas para sobrevivência e desenvolvimento.

\section{Espaços para a criação do conhecimento}

Nonaka, Toyama e Konno (2002) definem Ba como um espaço compartilhado para as relações emergentes, podendo ser:

- um espaço físico [um escritório, espaço de negócios (redes) etc.];

- virtual (um e-mail, uma teleconferência etc.);

- mental (das experiências compartilhadas, das ideias, dos ideais);
- ou uma múltipla combinação destas, sendo considerado um espaço compartilhado que serve de base para a criação de conhecimentos, seja este individual ou coletivo.

O que diferencia as empresas que utilizam o $B a$ das empresas que não o utilizam e dispõem apenas de interações normais humanas é o seu conceito de criação de conhecimento, uma vez que aquele não trata somente de simples interações entre os indivíduos, mas também disponibiliza uma plataforma para o conhecimento individual/coletivo avançado para a criação de conhecimentos.

Nonaka e Konno (1998) ainda propõem que o Ba seja construído de "informação necessária" à criação de conhecimentos, tanto individuais quanto coletivos, sendo as interações, desse modo, condicionadas por esse contexto rico em conhecimentos.

As trocas de dados, informação, opinião, colaboração e de uma mobilização sobre um projeto confrontado às necessidades e ao desconhecido convergem ao $\mathrm{Ba}$ dentro das organizações (FAYARD, 2003).

Segundo o mesmo autor, o $B a$ é fundamentalmente subjetivo e relacional, envolvendo os atores pelo fato de ser orientado pelo interesse e por não existirem fortes conflitos nos relacionamentos humanos.

Ci. Inf., Brasília, v. 38, n. 2, p. 96-109, maio/ago. 2009 
Desse modo, o papel dos dirigentes e executivos dentro das organizações é disponibilizar o $B a$ para a criação de conhecimentos e "gerenciar" a emergência do novo conhecimento. O Ba não vê a realidade por decreto, não é produzido pelo modelo do command and control próprio da gerência piramidal tradicional. Ao contrário, é ajustado por atores voluntários dentro de um ambiente energize and stimulate com atenção ao respeito mútuo.

\section{Os quarto $B a$ preconizados por Nonaka, Toyama e Konno (2002)}

Dentro do processo de criação de conhecimentos, Nonaka, Toyama e Konno (2002) apresentam quatro tipos de $B a$ : originating $b a$, dialoguing ba, systemizing ba exercising $b a$, a figura 3 descreve as características de todos os $B a$.

Originating ba é o espaço em que o conhecimento é originado por meio da interação face a face em que os indivíduos compartilham sentimentos, emoções, experiências e modelos mentais. É o primeiro ba no qual inicia o processo de criação de conhecimento e é associado ao processo de socialização do conhecimento tácito.

Experiências e habilidades transmitidas diretamente entre os indivíduos constituem a chave para converter conhecimento tácito em conhecimento tácito. É um espaço no qual emerge o amor, a confiança e o comprometimento, formando a base para a criação de conhecimento entre indivíduos. $\mathrm{O}$ originating ba é responsável pela emergência de ativos de conhecimento como habilidades, know-bow (NONAKA; TOYAMA; KONNO, 2002).

Dialoguing $b a$ é mais conscientemente construído em relação ao originating $b a$. Por meio do diálogo, indivíduos compartilham suas experiências e habilidades convertendo-as em termos e conceitos comuns. O dialoguing ba funciona como uma Ci. Inf., Brasília, v. 38, n. 2, p. 96-109, maio/ago. 2009
Type of Interaction

Individual

Collective

Originating Ba Dialoguing Ba

Virtual Exercising Ba $\quad$ Systemising Ba

plataforma para o processo de externalização do conhecimento em que o conhecimento tácito é tornado explícito. Ele promove a criação de ativos de conhecimento, como, por exemplo, conceitos de produtos, design e cenários futuros.

Systemizing $b a$ é definido como uma interação coletiva ou virtual e oferece um contexto para a combinação de novo conhecimento explícito gerado às bases de conhecimento existentes na organização. Nesta fase do processo as tecnologias de informação, como redes on-line, groupware etc., podem exercer um papel relevante para a sistematização do conhecimento explícito gerado. Ele é responsável pela emergência de ativos de conhecimento como database, documentos, especificações, manuais, patentes e licenças.

Exercising ba é definido como o espaço em que o conhecimento que foi socializado, combinado e sistematizado é novamente interpretado e internalizado pelo sistema cognitivo dos indivíduos. Neste tipo de ba ocorre a transformação de conhecimento explícito em conhecimento tácito, ou seja, o conhecimento criado é internalizado em forma de novos conceitos e práticas de trabalho. Nesses $B a$ são criados ativos de conhecimento como knowhow, rotinas organizacionais e novos padrões de comportamento. 


\section{ASPECTOS METODOLÓGICOS}

Nesse capítulo são apresentados os aspectos básicos que toda pesquisa científica requer para poder ser operacionalizada e após seu desenvolvimento oferecer conclusões e resultados válidos para acrescentar e consolidar novos conhecimentos.

A metodologia facilita e orienta o contexto e os caminhos necessários para poder executar adequadamente a coleta, tratamento e análise dos dados. De modo geral, esses caminhos incluem a escolha da taxonomia da pesquisa, em conformidade com o problema da pesquisa e os objetivos propostos, as escolhas das técnicas de coleta de dados na procura das fontes (primárias e secundárias) e a escolha dos métodos de tratamento e análise dos dados que permitam obter resultados e conclusões válidas para a pesquisa proposta.

\section{Tipo de estudo}

A pesquisa realizada se caracteriza como descritiva, pois se desejou fazer uma análise e reconhecimento das características dos fatos ou fenômenos pertinentes ao objeto de trabalho, apresentando um fenômeno ou circunstância, de acordo com um estudo feito em determinado espaço de tempo, no qual se obtiveram um levantamento de dados por tabulação e análise dos dados coletados (MARCONI; LAKATOS, 2003).

A pesquisa descritiva procura descobrir, com a precisão possível, qual a frequência com que um fenômeno ocorre, sua relação e conexão com outros, sua natureza e características, além de buscar conhecer as diversas situações e relações que ocorrem na vida social, política, econômica e demais aspectos do comportamento humano, tanto do indivíduo tomado isoladamente, quanto de grupos e comunidades mais complexas.

Outras fontes de informação também foram utilizadas para compor e fundamentar ainda mais o estudo. Entre elas se encontram as pesquisas bibliográficas por fontes secundárias, em leitura de livros, revistas, internet e documentos oficiais, e também fontes primárias, mediante contato direto por meio da pesquisa de campo, tabulação e análise dos dados obtidos.

O trabalho em questão também é do tipo estudo de caso. $\mathrm{O}$ estudo de caso é um dos vários modos de realizar uma pesquisa sólida. Em geral, estudos de casos se constituem na estratégia preferida quando o "como" e/ou o "por que" são as perguntas centrais, tendo o investigador um pequeno controle sobre os eventos, e quando o enfoque está em um fenômeno contemporâneo dentro de algum contexto de vida real.

Estudos de casos também podem ser classificados de várias maneiras, porém o presente trabalho refere-se a um "estudo de caso qualitativo".

\section{Universo e amostra}

O universo ou população é o conjunto de seres animados ou inanimados que apresenta pelo menos uma característica em comum, e a amostra é a porção ou parcela convenientemente selecionada de um universo (MARCONI; LAKATOS, 2003).

Assim, tem-se neste caso o universo como sendo os $100 \%$ dos estudantes do mestrado da Universidade Tecnológica do Paraná, Campus Ponta Grossa, formada por 79 alunos (referentes a alunos ingressos nos anos de 2007 e 2008), e a amostra foi por acessibilidade em que tais estudantes foram contatados e convidados a responder a tal instrumento de pesquisa por livre e espontânea vontade. Entre eles, 58 (73\%) dos estudantes responderam ao questionário.

A amostragem utilizada foi do tipo não probabilística, que se refere àquela em que a seleção dos elementos da população que são usados para compor a amostra depende ao menos em parte do julgamento do pesquisador ou do entrevistador no campo.

Ci. Inf., Brasília, v. 38, n. 2, p. 96-109, maio/ago. 2009 


\section{Técnica de coleta de dados}

$\mathrm{O}$ instrumento de pesquisa utilizado foi o questionário sugerido por Balestrin, Vargas e Fayard (2005) adaptado para aplicação junto às pessoas que compuseram a amostra. $\mathrm{O}$ mesmo é composto por sete questões que buscam identificar os quatro tipos de espaços organizacionais (originating $b a$, dialoguing ba, systemizing ba e exercising $b a$ ) e apontar seus benefícios para a criação de conhecimentos, de acordo com o quadro 1.

A importância do questionário, segundo Roesch (1999), é que ele não é apenas um formulário ou um conjunto de questões listadas sem muita reflexão. Ele explica que se trata de um instrumento de coleta de dados que busca mensurar alguma coisa, para tanto requer esforço intelectual anterior de planejamento, com base na conceituação do problema de pesquisa e do plano da pesquisa, bem como pesquisas exploratórias preliminares.

\section{A UNIVERSIDADE TECNOLÓGICA DO PARANÁ, CAMPUS PONTA GROSSA - UTFPR PG}

O Campus Ponta Grossa da Universidade Tecnológica Federal do Paraná (UTFPR) foi inaugurado dia 20 de dezembro de 1992 pelo ministro de Educação e Desporto Murilo de Avellar Hingel - portaria ${ }^{\circ}$ 1559, de 20 de outubro de 1992, na gestão do, então prefeito municipal, engenheiro Pedro Wosgrau Filho (UTFPR, 2008).

O Campus Ponta Grossa da UTFPR, antiga unidade do Centro Federal de Educação Tecnológica (CefetPR) iniciou suas atividades em 15 de março de 1993. As atividades educacionais iniciaram com a oferta dos cursos técnicos em alimentos e eletrônica.

A partir de 1995, passou a ofertar também curso técnico em mecânica. Em 1999, também pautada na LDBE, passou a ofertar, em nível de $3^{\circ}$ grau, os cursos superiores de tecnologia, uma forma

QUADRO 1

\section{Operacionalização das variáveis da pesquisa}

\begin{tabular}{|c|c|c|}
\hline $\begin{array}{l}\text { Elemento } \\
\text { conceitual }\end{array}$ & $\begin{array}{c}\text { Objetivo } \\
\text { da pesquisa }\end{array}$ & Variáveis da pesquisa \\
\hline $\begin{array}{c}\text { Contexto } \\
\text { capacitante }(b a) \\
\text { e criação do } \\
\text { conhecimento }\end{array}$ & $\begin{array}{c}\text { Identificar emergência } \\
\text { de contextos favoráveis } \\
\text { oo processo de criação de } \\
\text { conhecimento }\end{array}$ & $\begin{array}{l}\text { 1) Você como aluno (a) identifica algum espaço de criação do } \\
\text { conhecimento na UTFPR-PG e quais seus benefícios? } \\
\text { 2) Você como aluno (a) utiliza esses espaços de criação do } \\
\text { conhecimento com que frequência de tempo? } \\
\text { 3) Esses espaços identificados referem-se a espaços de criação do } \\
\text { conhecimento formalizados pela UTFPR-PG ou são informais? } \\
\text { 4) Quais os tipos e quantidades de espaços }(b a) \text { dedicados à } \\
\text { SOCIALIZAÇÃO do conhecimento na UTFPR-PG? } \\
\text { - Originating ba } \\
\text { 5) Quais os tipos e quantidades de espaços }(b a) \text { dedicados à } \\
\text { EXTERNALIZAÇÃO do conhecimento na UTFPR-PG? } \\
\text { - Dialoguing ba } \\
\text { 6) Quais os tipos e quantidades de espaços (ba) dedicados à } \\
\text { COMBINAÇÃO do conhecimento na UTFPR-PG? } \\
\text { - Systemizing ba } \\
\text { 7) Quais os tipos e quantidades de espaços }(b a) \text { dedicados à } \\
\text { INTERNALIZAÇÃO do conhecimento na UTFPR-PG? } \\
\text { - Exercising ba }\end{array}$ \\
\hline
\end{tabular}

Fonte: Balestrin, Vargas e Fayard (2005) [adaptado]. 
de graduação plena, com o objetivo de formar profissionais focados em tecnologia de ponta.

Atualmente, os cursos ofertados são Curso Superior de Tecnologia em Alimentos, Curso Superior de Tecnologia em Análise e Desenvolvimento de Sistemas, Curso Superior de Tecnologia em Automação, Curso Superior de Tecnologia em Fabricação Mecânica (UTFPR, 2008).

Aprovada no final de 2003, a pós-graduação stricto sensu iniciou suas atividades em 2004, com a oferta do Curso de Mestrado em Engenharia de Produção com duas linhas de concentração - gestão do conhecimento e inovação e gestão da produção e manutenção.

Em 2008 foi aprovado o mestrado profissional em ensino de ciência e tecnologia, com duas linhas de concentração: ciência e tecnologia no contexto do ensino-aprendizagem e construção do conhecimento no ensino de ciência e tecnologia.

\section{APRESENTAÇÃo E ANÁLISE DOS RESULTADOS}

A pesquisa junto aos alunos do mestrado em engenharia de produção da UTFPR-PG possibilitou observar o funcionamento de ambientes no que diz respeito à criação e disseminação do conhecimento.

As tabelas de 1 a 7 apresentam os resultados obtidos da pesquisa junto aos alunos da UTFPR-PG sobre a existência de $B a$ (físico, mental e virtual) e seus benefícios para a criação e disseminação dos conhecimentos.

Diante dos dados observados, é possível inferir que $100 \%$ dos respondentes identificam os espaços de criação do conhecimento na UTFPR-PG, sendo que $32 \%$ identificam algumas vezes, 50\% frequentemente e $17 \%$ sempre.

Alguns dos principais $B a$ identificados são reuniões, confraternizações, viagens e visitas, cursos, palestras etc.
TABELA 1

Identificação dos $B a$ pelos alunos na UTFPRPG

\begin{tabular}{l|r|r}
\hline $\begin{array}{c}\text { Identificação de algum espaço } \\
\text { de criação do conhecimento na } \\
\text { UTFPR-PG }\end{array}$ & TOTAL & $\%$ \\
\hline Nunca & 0 & 0 \\
Algumas vezes & 19 & 32 \\
Frequentemente & 29 & 50 \\
Sempre & 10 & 17 \\
TOTAL & 58 & 100 \\
\hline
\end{tabular}

Fonte: pesquisa de campo.

\section{TABELA 2}

Frequência de tempo da utilização dos $B a$ pelos alunos

\begin{tabular}{l|r|r}
\hline \multicolumn{1}{c|}{$\begin{array}{c}\text { Frequência de tempo da } \\
\text { utilização desses espaços de } \\
\text { criação do conhecimento }\end{array}$} & TOTAL & $\%$ \\
\hline Nunca & 4 & 7 \\
Algumas vezes (1 vez por semana) & 39 & 67 \\
Frequentemente (2 a 5 vezes por & 13 & 22 \\
semana) & 2 & 4 \\
Sempre (todos os dias) & 58 & 100 \\
TOTAL & & \\
\hline
\end{tabular}

Fonte: pesquisa de campo.

O principal benefício apontado pela identificação $\operatorname{dos} B a$ foi o compartilhamento de informações e de conhecimentos entre os alunos. As informações compartilhadas que mais trouxeram benefícios estão relacionadas à criação e desenvolvimento de artigos, seminários, qualificação e defesa da dissertação.

Já a maior dificuldade em relação à sua identificação está no fato de alguns $B a$ serem informais e/ou limitados a algum período de tempo e espaço.

Em relação à frequência de tempo da utilização dos $B a$ identificados, a tabela 2 apresenta os resultados. 
Diante dos dados observados é possível inferir que $93 \%$ dos respondentes utilizam algum dos Ba identificados na UTFPR-PG, sendo que $67 \%$ utilizam algumas vezes ( 1 vez por semana), $22 \%$ frequentemente ( 2 a 5 vezes por semana) e $4 \%$ sempre (todos os dias).

No entanto $7 \%$ dos alunos ainda não utilizam nenhuma vez por semana esses espaços. Os principais motivos da não utilização desses espaços por esses alunos são estar trabalhando, mas a empresa só liberar nos dias das aulas, residir em outras cidades e achar que os espaços não trazem informações importantes para o seu interesse.

Os principais benefícios para os $93 \%$ dos alunos que afirmam estar usando pelo menos uma vez na semana os $B a$ são o contato direto com os alunos e professores que ajudam a diminuir as dúvidas, aumentar as informações e consequentemente o conhecimento.

A tabela 3 ilustra os dados obtidos sobre a formalização ou informalização dos $B a$ no mestrado da UTFPR-PG.

Os dados demonstram que 58\% das respostas referem-se que os $B a$ são formalizados e $42 \%$ que não são formalizados. Esses dados evidenciam que existem ambientes formais, estruturados e visíveis aos alunos dentro do mestrado. A formalização de $B a$ pelo mestrado da UTFPR-PG proporciona mais interações entre os alunos e entre os professores, aumentando os níveis de confiança e criando redes de relacionamento.

Além desses, existem ambientes informais que os alunos também buscam para esclarecer suas dúvidas e trocar informações. Por ser informal, grande parte dos alunos sente-se mais solícita em colaborar com seus colegas e conversar com outros professores.

Já as tabelas de 4 a 7 estão relacionados aos tipos de $B a$ proposto pelo modelo de Nonaka, Toyama e Konno (2002). A tabela 4 refere-se ao primeiro Ba, a socialização do conhecimento.

Ci. Inf., Brasília, v. 38, n. 2, p. 96-109, maio/ago. 2009
TABELA 3

\begin{tabular}{l|c|c}
\hline $\begin{array}{c}\text { Ba formais ou informais na } \\
\text { UTFPR-PG (questão de múltipla } \\
\text { escolha) }\end{array}$ & TOTAL & $\%$ \\
\hline & & \\
\hline Formalizados & 40 & 58 \\
Não formalizados & 29 & 42 \\
TOTAL & 69 & 100 \\
\hline
\end{tabular}

Fonte: pesquisa de campo.

TABELA 4

\section{O Originating $b a$ - socialização do conhecimento}

\begin{tabular}{l|c|c}
\hline \multicolumn{1}{c|}{$\begin{array}{c}\text { Originating ba (questão de } \\
\text { múltipla escolha) }\end{array}$} & TOTAL & $\%$ \\
\hline Confraternizações & 28 & 36 \\
Visitas à indústria & 15 & 9 \\
Demais encontros informais & 36 & 45 \\
Outros & 0 & 0 \\
TOTAL & 79 & 100 \\
\hline
\end{tabular}

Fonte: pesquisa de campo.

As respostas obtidas sobre os $B a$ de socialização do conhecimento são as seguintes: $36 \%$ confraternizações, 19\% visitas à indústria, $45 \%$ a demais encontros informais.

As confraternizações, que normalmente são churrascos na associação dos servidores da UTFPRPG, são importantes momentos para solidificar as relações de confiança entre os alunos e professores e oportunizar, também, conversas informais sobre as oportunidades, os desafios e o futuro deles no mestrado.

As visitas à indústria sempre são inclusas no calendário dos mestrandos da UTFPR-PG. Elas dão aos alunos a oportunidade de conhecer outras realidades e refletir conjuntamente sobre seus temas e observar a sua aplicabilidade.

Os demais encontros informais são os espaços de socialização mais utilizados pelos alunos, estes são: 
os encontros na hora do almoço, no laboratório e na sala de estudo. Nesses ambientes informais os alunos tentam conversar usando metáforas, gestos, desenhos para explicar sua(s) ideia(s) para seu(s) colega(s).

Para o segundo espaço de criação do conhecimento, externalização, a tabela 5 apresenta os resultados.

As respostas obtidas sobre os $\mathrm{Ba}$ de externalização do conhecimento são $63 \%$ reuniões formais, $25 \%$ reuniões de planejamentos e $12 \%$ outros.

Reuniões formais. Esse $B a$ é sempre o primeiro contato do aluno com seu orientador. Eles acontecem e são espaços nos quais alunos e professores (principalmente os orientadores) compartilham suas experiências, emoções e sentimentos por meio da interação formal.

Reuniões de planejamento acontecem normalmente a cada semestre. O principal objetivo deste $B a$ são esclarecimentos de regras e procedimentos e do andamento do mestrado.

Os outros $B a$ de externalização identificados pelos alunos foram os seminários. Esses acontecem sempre em disciplinas do mestrado e têm por objetivo principal apresentar as ideias que serão desenvolvidas, que são combinadas com opiniões dos alunos e professores para elaboração de futuros artigos.

Do terceiro espaço de criação do conhecimento, combinação, a tabela 6 apresenta os resultados.

As respostas obtidas sobre os $B a$ de combinação do conhecimento são as seguintes: $76 \%$ comunicação eletrônica e $24 \%$ documentos formais.

Comunicação eletrônica. A utilização de recursos eletrônicos como e-mail e telefone também foi observada na dinâmica de criação e disseminação do conhecimento. Deve-se destacar que o uso dessas tecnologias representa papel importante para a comunicação e sistematização de conhecimento explícito.
TABELA 5

O Dialoguing ba - externalização do conhecimento

\begin{tabular}{l|r|r}
\hline \multicolumn{1}{c|}{$\begin{array}{c}\text { Dialoguing ba (questão de } \\
\text { múltipla escolha) }\end{array}$} & TOTAL & $\%$ \\
\hline Reuniões formais & 36 & 63 \\
Processo de tomada de decisão & 0 & 0 \\
coletiva & 14 & 25 \\
Reuniões de planejamento & 7 & 12 \\
Outros & 57 & 100 \\
\hline
\end{tabular}

Fonte: pesquisa de campo.

TABELA 6

Systemizing ba - combinação do conhecimento

\begin{tabular}{l|r|r}
\hline \multicolumn{1}{c|}{$\begin{array}{c}\text { Systemizing ba (questão de } \\
\text { múltipla escolha) }\end{array}$} & TOTAL & $\%$ \\
\hline Comunicação eletrônica & 45 & 76 \\
Documentos formais & 14 & 24 \\
Sistema de gestão compartilhado & 0 & 0 \\
Outros & 0 & 0 \\
TOTAL & 59 & 100,00 \\
\hline
\end{tabular}

Fonte: pesquisa de campo.

Os alunos utilizam os recursos eletrônicos para solicitar aos colegas informações sobre aulas e algum material (artigos, resumos etc.) que possam ser combinados com os que já estão disponíveis para sua pesquisa.

Documentos formais existem e sempre são solicitados pelos alunos. Vale destacar o uso das dissertações dos outros alunos dos mestrados, livros dos seus professores e artigos. Tais documentos estão disponíveis na biblioteca do campus ou na página do programa do mestrado na internet.

Do último espaço de criação do conhecimento, internalização, a tabela 7, a seguir, apresenta os resultados.

Ci. Inf., Brasília, v. 38, n. 2, p. 96-109, maio/ago. 2009 


\section{TABELA 7}

\section{O Dialoguing ba - externalização do conhecimento}

\begin{tabular}{l|r|r}
\hline \multicolumn{1}{c|}{$\begin{array}{c}\text { Exercising ba (questão de } \\
\text { múltipla escolha) }\end{array}$} & TOTAL & $\%$ \\
\hline $\begin{array}{l}\text { Novos conceitos } \\
\text { Novos produtos e/ou novos }\end{array}$ & 0 & 45 \\
serviços & 32 & 55 \\
Outras ações de aplicação do \\
$\begin{array}{l}\text { conhecimento } \\
\text { Outros }\end{array}$ & 0 & 0 \\
\hline
\end{tabular}

Fonte: pesquisa de campo.

As respostas obtidas sobre os $B a$ de internalização do conhecimento são as seguintes: $45 \%$ novos conceitos e $55 \%$ outras aplicações do conhecimento.

Novos conceitos. Esse $B a$ de internalização acontece de forma inicial quando os alunos conseguem aprovar os artigos que escreveram nas disciplinas. A partir dessa internalização de conhecimentos, eles aproveitam os novos conceitos para usar em suas dissertações.

Outras ações de aplicação do conhecimento. É o $B a$ de internalização mais usado. Os alunos recebem conhecimento por um período mínimo de um e máximo de dois anos, quando adquirem inúmeros conhecimentos que vão compor sua base cognitiva.

Diante dos novos conceitos eles vêem a possibilidade de inúmeras aplicações e vinculações com outras áreas do conhecimento, podendo utilizá-los também para futuro ingresso em empresas ou em um programa de doutorado.

$\mathrm{Na}$ figura 4, é retomado o modelo de Nonaka, Toyama e Konno (2002), na tentativa de classificar outros tipos de $B a$ (Originating Ba, Dialoguing $B a$, Systemizing $B$ a e Exercising Ba) identificados na dinâmica de criação de conhecimento pelos alunos do mestrado na UTFPR - PG.

Na UTFPR-PG, o Originating Ba é formado por diversos espaços, como, por exemplo, workshops, nos quais os alunos adquirem diretamente novas ideias advindas de renomados palestrantes. Existem também os grupos de estudos e a aula inaugu-ral, em que os alunos e professores compartilham suas experiências, emoções e sentimentos por meio da interação formal e informal.

O espaço de criação de conhecimento, denominado Dialoguing ba, ocorre também nos corredores e no café. Essas atividades informais na UTFPR-PG servem de plataforma para os alunos, por meio do diálogo e reflexão coletiva, compartilharem suas

FIGURA 4

Classificação dos diferentes tipos de $b a$

\begin{tabular}{|c|c|c|c|}
\hline & Tácito & Tácito & \\
\hline \multirow{4}{*}{ 尊 } & Originating $b a$ & Dialoguing $b a$ & \multirow{7}{*}{ 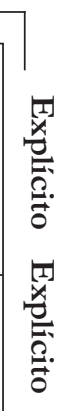 } \\
\hline & Workshops & & \\
\hline & Grupo de estudo & Corredores e café & \\
\hline & Aula inaugural & Participação em congressos & \\
\hline \multirow{3}{*}{ 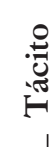 } & Exercising $b a$ & Systemizing ba & \\
\hline & Publicação de artigos & Campus virtual & \\
\hline & Dissertação & Biblioteca & \\
\hline
\end{tabular}

Fonte: pesquisa de campo 
ideias, experiências, modelos mentais, convertendoos em termos e conceitos comuns.

O Systemizing ba é um contexto que permite aos alunos integrar novos conhecimentos explícitos aos conhecimentos existentes. Na UTFPR-PG, há utilização da biblioteca e do campus virtual (ferramenta que proporciona troca de documentos e interação direta via chat ou indireta por e-mail). No entanto, a ferramenta campus virtual está em fase de implementação, podendo algumas das suas funcionalidades não estar ainda acessíveis para os alunos.

A última fase do processo de criação do conhecimento - Exercising $B a$-, na qual o conhecimento é internalizado e aplicado em termos de novas práticas acadêmicas, apresentou resultados no mestrado da UTFPR-PG, como, por exemplo, a publicação de artigos e dissertação.

\section{CONSIDERAÇÕES FINAIS}

O presente estudo tem como principal objetivo buscar a compreensão da dinâmica de criação e utilização do conhecimento. A perspectiva de análise refere-se ao contexto ou espaço físico, virtual e mental, dentro do qual o conhecimento é criado e utilizado, que é denominado pela expressão japonesa Ba.

Partindo do princípio das dificuldades de criação do conhecimento, torna-se necessária a identificação dos meios que as organizações devem utilizar para conseguir "gerenciar" o conhecimento que é criado e potencializá-lo de forma que dele se criem inovações. Em função disso, a formação dos $B a$ organizacionais constitui mecanismos capazes de potencializar a criação e disseminação do conhecimento.

Os resultados demonstram que o uso de $B a$ proporciona para o mestrado em engenharia de produção da Universidade Federal Tecnológica do Paraná, campus Ponta Grossa, condições favoráveis a uma dinâmica ativa aos processos de criação e ampliação de seus conhecimentos.
O surgimento de alguns espaços formais e informais proporciona aos alunos possibilidades de compartilhamento de suas habilidades, experiências, emoções e know-how, em razão da comunicação face a face e compartilhamento e disseminação de conhecimento tácito, sendo que as características desse tipo de conhecimento são consideradas essenciais para a sustentabilidade das vantagens competitivas.

Deve-se destacar que o conceito de Ba é de relevante importância para se entender a verdadeira natureza do processo de criação de conhecimento junto às organizações japonesas. No entanto, as teorizações e evidências apresentadas não têm a pretensão de ser uma teoria definitiva, e sim visam a estimular o debate e a crítica sobre os conceitos de criação de conhecimento e $B a$ junto à comunidade acadêmica brasileira.

\section{Agradecimentos}

Os autores agradecem à Coordenação de Aperfeiçoamento de Pessoal de Nível Superior (Capes) pelo financiamento da pesquisa.

Grupo de estudo Bioprodução.

Artigo submetido em 11/12/2008 e aceito em 15/07/2009.

\section{REFERÊNCIAS}

BARCLAY, R. U.; MURRAY, P. What is Knowledge Management. In:__ A Knowledge Praxis. [S.l.]: [s.n.], 1997.

BALESTRIN, A.; VARGAS, L. M.; FAYARD, P. Ampliação interorganizacional do conhecimento: o caso das redes de cooperação. Revista eletrônica de administração, v. 11, n. 1, jan./ fev. 2005.

CHOO, C. W. A organização do conbecimento: como as organizações usam a informação para criar significado, construir conhecimento e tomar decisões. São Paulo: Senac São Paulo, 2003.

FAYARD, P. Le concept de ba dans la voie japonaise de la création du savoir: rapports d'Ambassade. [S.I.] : Ambassade de France au Japon, 2003.

LEONARD-BARTON, D. Wellsprings of Knowledge. Boston: Harvard Business School Press, 1995.

Ci. Inf., Brasília, v. 38, n. 2, p. 96-109, maio/ago. 2009 
A percepção dos alunos do mestrado em engenharia de produção sobre a existência de ambientes de criação do

NONAKA, I.; TAKEUCHI, H. The knowledge-creating company: how Japanese companies create the dynamics of innovation. New York: Oxford University Press, 1997.

MARCONI, M. A.; LAKATOS, E. M. Técnicas de pesquisa. 5. ed. São Paulo: Atlas, 2003.

NONAKA, I.; KONNO, N. The concept of "Ba": building a foundation for knowledge creation. California Management Review, v. 40, n. 3 , Spring 1998.

NONAKA, I.; TOYAMA, R. E.; KONNO, N. SECI, ba and leadership: a unified model of dynamic knowledge creation. In:__. Managing knowledge an essential reader. London: Sage Publications, 2002.

PERROW, C. Small-Firm networks. In: NOHRIA, N.; ECCLES, R. Networks and organizations: structure, form and action. Boston: Harvard Business School Press, 1992.

PROBST, G.; RAUB, S.; ROMHARDT, K. Gestão do conbecimento: os elementos construtivos do sucesso. Tradução Maria Adelaide Carpigiani. Porto Alegre: Bookman, 2002. Tradução de: Managing knowledge: building blocks for success.
ROESCH, S. M. A. Projetos de estágio e de pesquisa em administração: guias para estágios, trabalhos de conclusão, dissertações e estudos de casos. 2. ed. São Paulo: Atlas, 1999.

SABEL, C. Moebius-strip organizations and open labor markets: some consequences of the reintegration of conception and execution in a volatile economy. In: COLEMAN, J.; BOURDIEU, P. Social theory for a changing society. Boulder: Westview Press, 1991.

UNIVERSIDADE TECNOLOGICA FEDERAL DO PARANÁ. História do Campus Ponta Grossa, UTFPR-PG. Disponível em : <http:// www.pg.cefetpr.br/?pag_id=6>. Acesso em: abr. 2008.

VON KROGH, G.; ICHIJO, K.; NONAKA, I. Facilitando a criação do conbecimento: reinventando a empresa com o poder da inovação contínua. Rio de Janeiro: Campus, 2001.

WIIG, K. M. Knowledge management foundations: thinking about thinking: how people and organizations create, represent, and use knowledge. Arlington, Texas: Schema Press, 1993. 